\title{
Viabilidade da equação do arco da parábola no cálculo do perímetro molhado na irrigação por sulco ${ }^{1}$
}

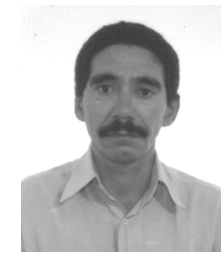

Roberto V. Pordeus ${ }^{2}$, Francisco de Souza ${ }^{3} \&$ Carlos A.V. de Azevedo ${ }^{4}$

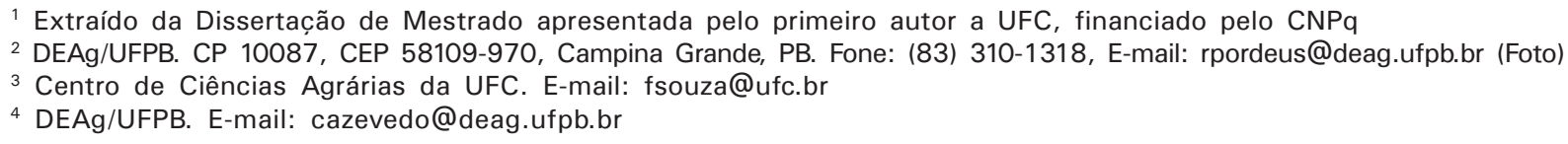

Protocolo $080-10 / 07 / 2000$

\begin{abstract}
Resumo: Neste trabalho, estudou-se a viabilidade da equação do arco da parábola no cálculo do perímetro molhado, para fins de avaliação do desempenho da irrigação por sulco. Os valores do perímetro molhado estimados pela equação proposta, foram avaliados mediante comparação com resultados obtidos a partir de outras equações apresentadas na literatura e com dados de campo. Os resultados mostraram que a equação do arco da parábola é viável no cálculo do perímetro molhado, na irrigação por sulco.
\end{abstract}

Palavras-chave: irrigação, sulco, modelo matemático

\section{Feasibility of the parabolic arc equation for calculating the wetted perimeter in furrow irrigation}

\begin{abstract}
For purposes of evaluation of performance of furrow irrigation, this research verified the feasibility of the parabolic arc equation for calculating the wetted perimeter. The values of the wetted perimeter estimated by the proposed equation were evaluated by comparison with results obtained from others equations presented in the literature and with the field data. The results showed that the parabolic arc equation is feasible for calculating the wetted perimeter in furrow irrigation.
\end{abstract}

Key words: furrow irrigation, wetted perimeter, performance

\section{INTRODUÇÃO}

Nas últimas décadas, vários modelos matemáticos foram apresentados para simular a irrigação por superfície, sendo classificados em hidrodinâmicos, zero-inércia, ondas cinemáticas, e os mais simplificados baseados no balanço de volume d'água. Esses modelos permitem uma análise rápida e econômica dos parâmetros da irrigação por superfície. Segundo Souza (1983) a análise da irrigação por sulco é mais difícil que a por faixas, devido à complexidade introduzida na forma do sulco e sua relação com a taxa de infiltração. Azevedo (1992) desenvolveu o modelo SIRTOM que é um sistema de checagem e controle capaz de predizer as características de infiltração num tempo real e promover ajustes, em tempo real, na operação do evento de irrigação, melhorando o desempenho do sistema.

A infiltração em sulcos pode ocorrer em duas ou três direções que, conforme Philip (1966), apud Fok (1988) seu estudo usa análise matemática avançada. Bernardo (1989) a forma geométrica dos sulcos, incluindo-se a profundidade, é muito importante na eficiência e na adequação da irrigação. A profundidade da lâmina d'água no sulco é influenciada pela forma do sulco, rugosidade e vazão de entrada; esta deverá ser suficiente para infiltrar a lâmina requerida no final do sulco.

De acordo com o SCS-USDA (1980) a água infiltrada por unidade de comprimento de sulco é diretamente proporcional à superfície do solo em contato com a água, sendo que esta superfície depende do perímetro molhado. A infiltração na irrigação por sulco ocorre tanto na direção horizontal como na vertical, em contraste com a irrigação por inundação e por aspersão, em que só ocorre infiltração na direção vertical. Ao perímetro molhado $\left(\mathrm{P}_{\mathrm{m}}\right)$ encontrado, o SCS-USDA (1989) adicionou uma constante empírica para considerar a infiltração horizontal causada pelo gradiente do potencial total da água no solo, este é chamado perímetro molhado ajustado. A equação apresentada para sulcos e corrugações, é a seguinte:

$$
\mathrm{P}_{\mathrm{m}}=0,265\left(\mathrm{Qn} / \mathrm{S}_{0}^{0,5}\right)^{0,425}+0,227
$$

em que $\mathrm{P}_{\mathrm{m}}$ é o perímetro molhado ajustado, $\mathrm{m}$; $\mathrm{Q}$ é a vazão, $\mathrm{L} \mathrm{s}^{-1}$; $\mathrm{S}_{0}$ é o gradiente hidráulico, $\mathrm{m} \mathrm{m}^{-1}$; e $\mathrm{n}$ é o coeficiente de rugosidade de Manning. 
A menor seção transversal de escoamento do sulco confere algumas características hidráulicas que as diferenciam das faixas. Dentre estas características encontram-se as equações necessárias à caracterização dessa geometria, as quais introduzem complicações adicionais ao tratamento hidráulico do processo de escoamento. Segundo Scaloppi (1986) a geometria da seção de escoamento tem grande influência na taxa de avanço da água na superfície, através de seus efeitos sobre a área de infiltração, resistência ao escoamento e capacidade de armazenamento de água. De acordo com Azevedo (1993), a baixa eficiência de aplicação dos projetos de irrigação superficial se deve a um inadequado dimensionamento e um manejo inapropriado.

De acordo com Olitta (1978) a forma dos sulcos pode exercer influência considerável na eficiência da irrigação. A seção transversal deve ser suficiente para armazenar a quantidade de água necessária a uma distribuição uniforme ao longo do comprimento do sulco. O formato depende principalmente do implemento agrícola utilizado, da cultura e do tipo de solo, podendo aproximar-se da forma triangular, retangular e trapezoidal. Devido ao movimento da água, com o tempo essas geometrias tendem a uma forma parabólica, principalmente os sulcos de seção triangular e trapezoidal. Carvalho et al. (1995) analisaram num solo aluvial, a influência do perfil geométrico do sulco, no desempenho da irrigação e constataram que nos perfis parabólicos, houve uma tendência de se obter maiores eficiência de aplicação (Ea) em relação aos perfis triangular e trapezoidal.

Os sulcos apresentam dimensões variadas nas seções transversal e longitudinal, em função da textura do solo e da cultura explorada. Para os solos com baixa velocidade de infiltração, recomendam-se sulcos raso e largo, que geralmente apresentam a forma de U, enquanto para solos com alta velocidade de infiltração, recomenda-se sulco estreito e mais profundo, geralmente em forma de V. Essas geometrias tendem à forma parabólica. Souza (1981) e Levien (1985) afirmam que em sulcos de forma parabólica, quando a profundidade, $y$, for muito pequena em relação à largura, o perímetro molhado $\left(\mathrm{P}_{\mathrm{m}}\right)$ será aproximadamente igual à largura da superfície livre da água (B) ou seja:

$$
\mathrm{P}_{\mathrm{m}}=\mathrm{B}=\mathrm{C} \mathrm{y}^{\mathrm{M}}
$$

Segundo Scaloppi (1986) os valores de contasntes C e M, normalmente observados na prática, impedem que o perímetro molhado no sulco $\left(\mathrm{P}_{\mathrm{m}}\right)$ seja calculado por soluções diretas; acrescenta, ainda, que na maioria das situações as soluções são aproximadas por integração numérica o que desaconselha sua determinação sem a utilização de recursos que nem sempre se encontram disponíveis. Deve-se, então, encontrar soluções aproximadas; seria o caso, por exemplo, assumir o perímetro molhado igual a B. Evidentemente, a grandeza do erro decorrente dessa aproximação será proporcional às diferenças entre o valor de $\mathrm{M}$, pois quanto menor o valor de $\mathrm{M}$, maior a aproximação entre $\mathrm{B}$ e $\mathrm{P}_{\mathrm{m}}$, para qualquer valor de $\mathrm{y}$.

Souza (1981) desenvolveu um modelo hidrodinâmico da irrigação por sulco, no qual considera o sulco de forma parabólica definida pela Eq. 2 e calcula o perímetro molhado por integração numérica, pela seguinte expressão:

$$
P_{m}=2 \int\left[1+(M B / 2 y)^{2}\right]^{\frac{1}{2}} d y
$$

Amim (1985) recomenda que as medições da largura da superfície livre da água (B) devem ter o seguinte procedimento: quando a frente de avanço atingir a $1^{\mathrm{a}}$ estação, faz-se a medição de B na estação "zero"; quando atingir a $2^{\mathrm{a}}$ estação, fazem-se as medições na $1^{\mathrm{a}}$ e na estação "zero", e assim sucessivamente.

Quando a lâmina d'água é considerada pequena, os modelos tradicionais da literatura funcionam bem no cálculo do perímetro molhado, podendo-se considerar a largura da superfície livre de água igual $\mathrm{a} \mathrm{P}_{\mathrm{m}}$; no entanto, à medida que a lâmina d'água cresce, esses modelos tornam-se praticamente inválidos; assim sendo, existem grandes dificuldades de determinação da superfície de contato da água com o solo, isto é, do perímetro molhado em sulco, para lâminas consideradas não muito pequenas, motivo pelo qual se procurou, com este trabalho, desenvolver uma equação que melhor representasse a superfície de contato da água com a superfície do solo, a qual se baseou na geometria da parábola.

\section{MATERIAL E MÉTODOS}

Para se analisar a viabilidade da equação do arco da parábola no cálculo da superfície de contato da água com o solo, perímetro molhado, assumiu-se que a superfície livre da água (B) é definida pela Eq. 2, em que C e M são constantes empíricas e y é a profundidade do escoamento no ponto central do sulco. Para a forma parabólica o valor de $\mathrm{M}$ deve ser maior que $0 \mathrm{e}$ menor que 1 .

Depois de definida a forma da seção transversal do sulco, a preocupação seguinte é se conhecer as relações métricas envolvidas com suas dimensões e sua área, perímetro molhado e raio hidráulico; enfim, considerando-se que a forma do sulco é parabólica, determinou-se a seguinte equação para o perímetro molhado.

Devido à complexidade da geometria do sulco foram consideradas, neste trabalho, três equações para o cálculo do perímetro molhado.

Para o primeiro caso, $\mathrm{P}_{1}$, considerou-se o perímetro molhado igual à superfície livre da água dado pela Eq. (2). Conforme alguns autores, quando a lâmina d'água y é muito pequena, o perímetro molhado pode ser aproximado por B; no segundo caso, $\mathrm{P}_{2}$, o perímetro molhado foi calculado pela Eq. (1) e para o terceiro caso, $\mathrm{P}_{3}$, como o sulco é de forma parabólica, pode-se determinar o comprimento do arco da parábola e assumir que este é igual ao perímetro molhado do sulco. Seja a Figura 1 para a parábola:

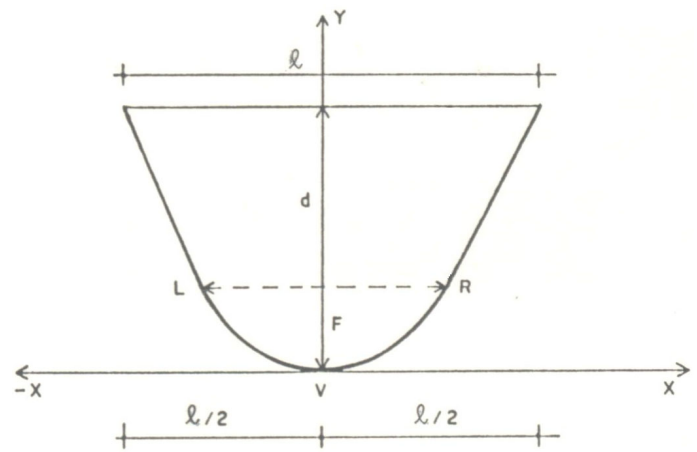

Figura 1. Ilustração do arco da parábola 
sendo:

$$
\mathrm{x}^{2}=\mathrm{ay}
$$

onde a é a corda focal mínima da parábola.

O comprimento do arco, s, pode ser calculado como:

$$
s=\int_{0}^{1 / 2} \sqrt{1+\left(\frac{d y}{d x}\right)^{2}} d x
$$

como ay $=\mathrm{x}^{2}$, tem-se que $\mathrm{y}^{\prime}=2 \mathrm{x} / \mathrm{a}$. Utilizando identidade trigonométrica, para resolver a seguinte função:

$$
\sec \theta=\sqrt{1+\operatorname{tg}^{2} \vartheta}
$$

no intervalo $-\theta / 2<\theta<\theta / 2$, e se considerando $u=\operatorname{tg} \theta=2 x / \mathrm{a}$, cuja derivada de $\mathrm{x}(\mathrm{d} x)$ é igual metade de a vezes a secante ao quadrado de $\theta$ vezes a derivada de $\theta, d \theta$, obtém-se a seguinte equação:

$$
S=\int_{0}^{1 / 2} \sqrt{1+\left(\frac{2 x}{a}\right)^{2}} d x=\frac{a}{2} \int \sec ^{3} \theta d \theta
$$

Integrando a Eq. (7) por partes e admitindo-se os dois lados do arco, obtém-se a seguinte equação:

$$
\mathrm{S}=\frac{1}{2} \sqrt{\ell^{2}+16 \mathrm{~d}^{2}}+\frac{\ell^{2}}{8 \mathrm{~d}} \operatorname{Ln}\left(\frac{4 \mathrm{~d}+\sqrt{\ell^{2}+16 \mathrm{~d}^{2}}}{\ell}\right)
$$

Considerando-se a profundidade da lâmina d'água na cabeceira do sulco $\mathrm{y}_{\mathrm{n}}=\mathrm{d}$ e a largura $\mathrm{l}=\mathrm{B}$, tem-se:

$\mathrm{S}=\mathrm{P}_{\mathrm{m}}=\frac{1}{2} \sqrt{\mathrm{B}^{2}+16 \mathrm{y}_{\mathrm{n}}{ }^{2}}+\frac{\mathrm{B}^{2}}{8 \mathrm{y}_{\mathrm{n}}} \operatorname{Ln}\left(\frac{4 \mathrm{y}_{\mathrm{n}}+\sqrt{\mathrm{B}^{2}+16 \mathrm{y}_{\mathrm{n}}{ }^{2}}}{\mathrm{~B}}\right)(9)$

em que $\mathrm{y}_{\mathrm{n}}$ é a lâmina d'água normal na cabeceira do sulco, determinado pela seguinte equação.

$$
\mathrm{y}_{\mathrm{n}}(\mathrm{Q})=\left\{\frac{\mathrm{Qn}}{\mathrm{C}_{\mathrm{u}} \mathrm{S}_{0}{ }^{0,5}} \frac{(\mathrm{M}+1)^{5 / 3}}{\mathrm{C}}\right\}^{\frac{3}{3 \mathrm{M}+5}}
$$

em que:

$$
\begin{array}{ll}
\mathrm{y}_{\mathrm{n}}(\mathrm{Q}) & \text { - profundidade normal, } \mathrm{m} \\
\mathrm{Q} & \text { - vazão de entrada, } \mathrm{m}^{3} \mathrm{~s}^{-1}
\end{array}
$$

A viabilidade da Eq. (9) pode ser observada comparando-se seus resultados com aqueles de medições de campo e obtidos a partir de equações apresentadas na literatura e, ainda, extraídos de modelos matemáticos de simulação da irrigação superficial. Para tanto, o valor do volume infiltrado encontrado a partir da equação determinada neste trabalho, foi comparado com valores obtidos a partir das equações sugeridas por Levien (1985) e SCS-USDA (1989) com dados de campo e com o valor encontrado por Souza (1981) através de seu modelo hidrodinâmico. Utilizaram-se os dados de campo obtidos em experimentos realizados por Ramsey (1976) e Karmeli (Horticulture, 1979). Esses dados foram escolhidos pela sua acuracidade. No desenvolvimento deste trabalho utilizou-se, ainda, o modelo matemático algébrico desenvolvido por Levien (1985) para realizar cálculos de parâmetros hidráulicos inerentes à avaliação da irrigação por sulco.

No cálculo do avanço utilizou-se a equação do balanço volumétrico para sulcos apresentados por Souza (1981) expressa da seguinte forma:

$$
\mathrm{x}_{\mathrm{a}}=\frac{\mathrm{Qt}}{\mathrm{A}_{0} \mathrm{r}_{\mathrm{y}}+\mathrm{A}_{\mathrm{zo}} \mathrm{r}_{\mathrm{z}}}
$$

em que:

$\mathrm{x}_{\mathrm{a}} \quad$ - distância do avanço da água para um intervalo de tempo, $\mathrm{t}$

$r_{y}$ e $r_{z}$ - fatores de forma superficial e sub-superficial, considerados constantes e iguais a 0,75 e 0,80 respectivamente,

$A_{0}$ e $A_{z 0}$ - seção transversal de fluxo na entrada e saída do sulco, dados pelas seguintes equações:

$$
\begin{gathered}
A_{0}=\frac{B y_{n}}{(M+1)} \\
A_{Z 0}=P_{M} Z(t)
\end{gathered}
$$

em que: $Z(t)$ lâmina d'água infiltrada função de $t$, dada pela seguinte equação:

$$
\mathrm{Z}(\mathrm{t})=\mathrm{kt}^{\mathrm{a}}
$$

sendo $\mathrm{k}$ e a constantes para determinado nível de umidade e $\mathrm{t}$ tempo de infiltração.

O tempo de recessão considerado foi o tempo de recessão constante e superior ao tempo de aplicação, utilizado por Levien (1985) considerada como a recessão na cabeceira do sulco, dado pela seguinte equação:

$$
\mathrm{t}_{\mathrm{r}}=\mathrm{t}_{\mathrm{co}}+\frac{\mathrm{A}_{0}}{(\mathrm{M}+1)} \frac{\mathrm{L}}{\mathrm{Q}_{0}}
$$

donde:

$\mathrm{t}_{\mathrm{r}} \quad$ - recessão constante e superior ao tempo de aplicação, min

L - comprimento do sulco, $\mathrm{m}$ 
$\mathrm{O}$ volume infiltrado, $\mathrm{V}_{\mathrm{z}}$, foi obtido pela seguinte equação:

$$
\mathrm{V}_{\mathrm{Z}}=\frac{\mathrm{Z}\left(\mathrm{t}_{\mathrm{r}}\right)+\mathrm{Z}\left(\mathrm{t}_{\mathrm{r}}-\mathrm{t}_{\mathrm{av}}\right)}{2} \mathrm{P}_{\mathrm{m}} \mathrm{L}
$$

donde $\mathrm{t}_{\mathrm{av}}$ é tempo de avanço, min.

Para a análise da viabilidade da equação do arco da parábola no cálculo da superfície de contato da água com o solo, perímetro molhado e sua influência sobre o avanço da água, lâmina infiltrada e, consequentemente, volume infiltrado, utilizaram-se dados de campo (Tabela 1), obtidos em experimentos realizados por Ramsey (1976) e Karmeli (1979), utilizou-se também como parâmetro de referência o volume infiltrado calculado pelo modelo hidrodinâmico, com dados do segundo experimento. Nas Figuras 2 e 3, são apresentados as curvas de avanço, de recessão e as lâminas infiltradas calculadas usando-se os três valores de $\mathrm{P}_{\mathrm{m}}$ (Tabela 2). Nestas Figuras são apresentados também, os resultados obtidos no campo e com o modelo hidrodinâmico considerado como padrão pela sua precisão.

\section{RESULTADOS E DISCUSSÃO}

$\mathrm{Na}$ Tabela 2, estão apresentados os valores de 0,273 e $0,258 \mathrm{~m}$ para $P_{1}$ e 0,482 e $0,379 \mathrm{~m}$ para $P_{2}$, enquanto $P_{3}$ foi de 0,294 e $0,262 \mathrm{~m}$. Verifica-se que os valores de $P_{1}$ e $P_{3}$ foram muito próximos, o que não se verificou com $\mathrm{P}_{2}$ observando-se, portanto, para a menor lâmina “yn” (Karmelli), $\mathrm{P}_{3}$ tende a se aproximar de
Tabela 1. Dados de campo utilizados na análise da irrigação por sulco

\begin{tabular}{lcc}
\hline \multirow{2}{*}{ Parâmetros } & \multicolumn{2}{c}{ Experimentos } \\
\cline { 2 - 3 } $\mathrm{Q}_{\mathrm{o}}\left(\mathrm{L} \mathrm{s}^{-1}\right)$ & Ramsey $(1976)$ & Karmelli (1979) \\
$\mathrm{S}_{\mathrm{o}}\left(\mathrm{m} \mathrm{m}^{-1}\right)$ & 1,33 & 0,81 \\
$\mathrm{~L}(\mathrm{~m})$ & 0,001032 & 0,0036 \\
$\mathrm{t}_{\mathrm{co}}(\mathrm{min})$ & 100 & 175 \\
$\mathrm{k}\left(\mathrm{cm}\left(\mathrm{min}^{\mathrm{a}}\right)^{-1}\right)$ & 208 & 202 \\
$\mathrm{a}$ & 0,98 & 0,77 \\
$\mathrm{n}$ & 0,50 & 0,55 \\
$\mathrm{E}(\mathrm{m})$ & 0,022 & 0,020 \\
$\mathrm{C}$ & 1,0 & 1,12 \\
$\mathrm{M}$ & 1,091 & 0,61 \\
\end{tabular}

$\mathrm{P}_{1}$, superando em $1,5 \%$, enquanto para a maior lâmina esta diferença atingiu o valor de $7 \%$, possivelmente, pelo fato da equação de $\mathrm{P}_{1}$ não estar representando bem o perímetro molhado, uma vez que, a maior aproximação entre a superfície livre da água (B) e o $\mathrm{P}_{1}$, ocorre para menor valor de M. Quando " $\mathrm{y}_{\mathrm{n}}$ " for muito pequeno em relação a $\mathrm{B}$, o perímetro molhado é aproximadamente igual a largura da superfície livre da água (B). Com estes resultados constatou-se que com o decréscimo da lâmina, os valores de $\mathrm{P}_{3}$ e $\mathrm{P}_{1}$ se assemelham, podendo, portanto, o perímetro molhado ser representado por ambos.

No entanto, quando a lâmina " $\mathrm{y}_{\mathrm{n}}$ " cresce, independente do valor de $\mathrm{M}$, o perímetro molhado é melhor representado por $\mathrm{P}_{3}$, pois o aumento da lâmina " $\mathrm{y}_{\mathrm{n}}$ ", faz com que a medida da superfície de contato da água com o solo torna-se superior a superfície livre da água (B). A principal condição para $\mathrm{P}_{1}$ representar o perímetro molhado é que a superfície livre da

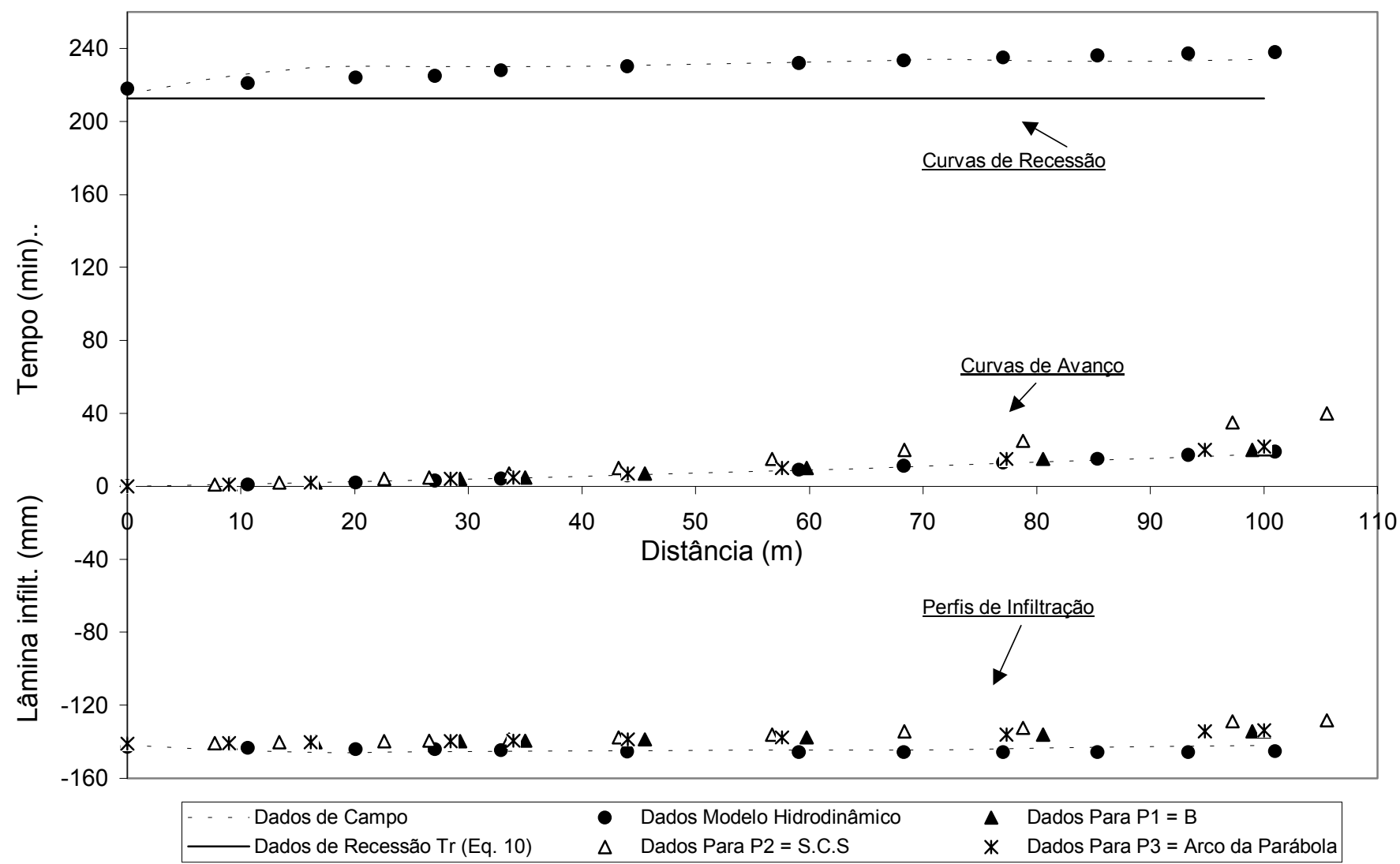

Figura 2. Curvas de avanço, recessão e perfil de infiltração, calculados com os perímetros $\mathrm{P}_{1}, \mathrm{P}_{2}$ e $\mathrm{P}_{3}$, comparados com dados de campo (Ramsey, 1976) e com o modelo hidrodinâmico, sulco com comprimento de $100 \mathrm{~m}$ 


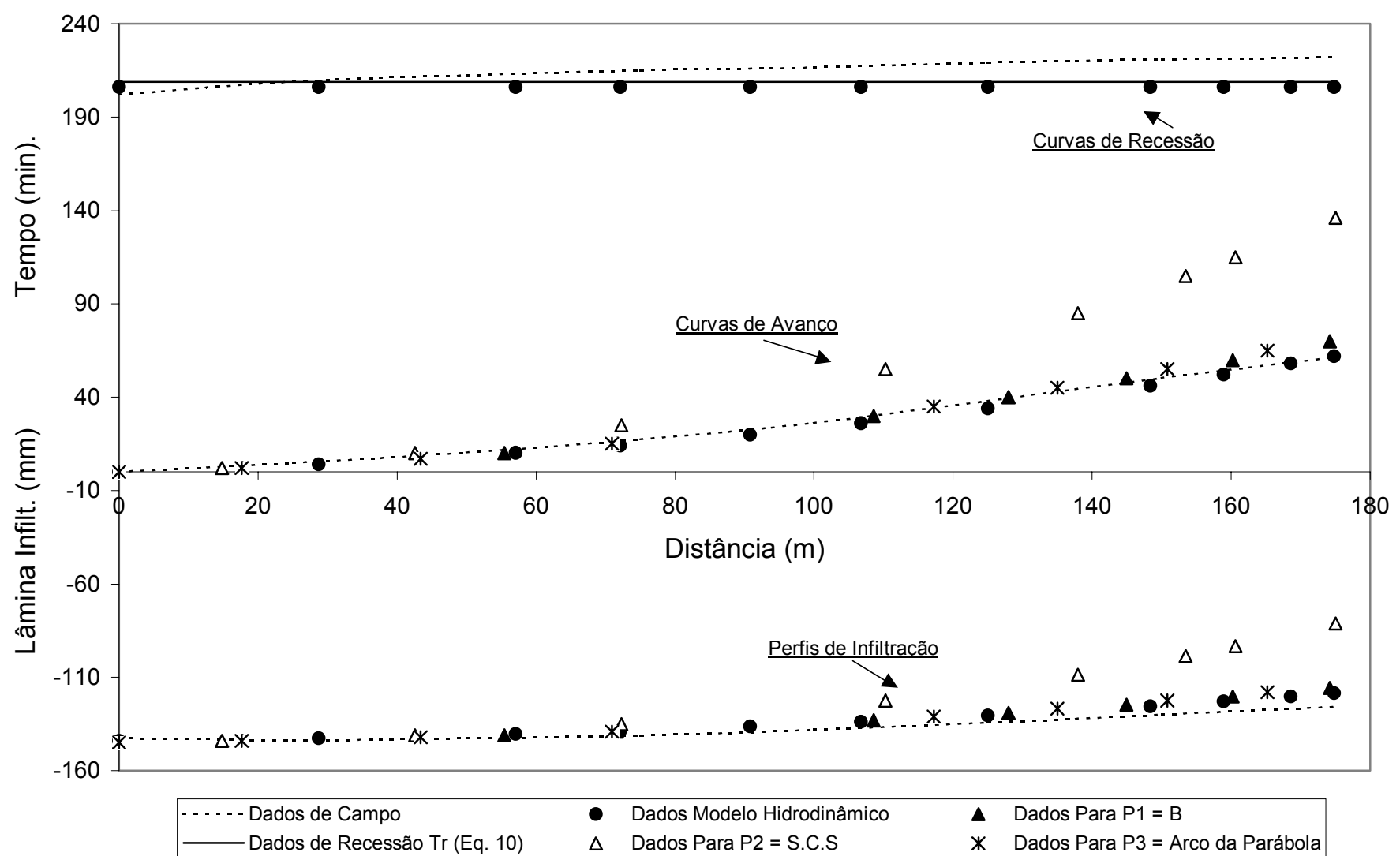

Figura 3. Curvas de avanço, recessão e perfil de infiltração, calculados com os perímetros $\mathrm{P}_{1}, \mathrm{P}_{2}$ e $\mathrm{P}_{3}$, comparados com dados de campo (Karmeli, 1979) e com o modelo hidrodinâmico, sulco com comprimento de 175 m

Tabela 2. Valores do perímetro molhado, $\mathrm{P}_{\mathrm{m}}$, encontrados usando-se as diferentes formas de cálculo

\begin{tabular}{lcccc}
\hline \multirow{2}{*}{ Experimentos } & $\mathrm{y}_{\mathrm{n}}$ & $\mathrm{P}_{1}$ & $\mathrm{P}_{2}$ & $\mathrm{P}_{3}$ \\
\cline { 2 - 5 } & \multicolumn{4}{c}{$(\mathrm{m})$} \\
\hline 1. Ramsey & 0,05647 & 0,273 & 0,482 & 0,294 \\
2. Karmeli & 0,02368 & 0,258 & 0,379 & 0,262 \\
\hline
\end{tabular}

água seja aproximadamente igual a superfície de contato da água com o solo, nesse caso, a equação que representa $\mathrm{P}_{3}$ pode ser utilizado para qualquer lâmina.

Quando comparado $\mathrm{P}_{3}$ com $\mathrm{P}_{2}$, verifica-se que o valor de $\mathrm{P}_{3}$ é inferior em 39 e $30 \%$ para maior e menor lâmina respectivamente. Neste caso, verifica-se que esta diferença deve-se provavelmente ao fato de $\mathrm{P}_{2}$ receber um incremento no valor de 0,227 , para corrigir a infiltração horizontal, em condições normais de irrigação, haja visto, que no caso em estudo trabalhou-se com lâminas muito pequenas, onde o valor da superfície de contato da água com o solo se aproxima da largura da superfície livre da água.

Observa-se, nas Figuras 2 e 3, que as curvas de avanço apresentam, quando se utilizam os perímetros $\mathrm{P}_{1}$ e $\mathrm{P}_{3}$; valores muito próximos dos obtidos em campo e com os valores obtidos se utilizando do modelo hidrodinâmico, como referência. Enquanto a curva de avanço apresenta, quando se utiliza $\mathrm{P}_{2}$, valores superiores aos dados de campo, verifica-se portanto, que na equação de avanço, tanto $\mathrm{P}_{1}$ quanto $\mathrm{P}_{3}$ representam bem os dados de campo quando utilizados na equação de avanço, enquanto que, com $\mathrm{P}_{2} \mathrm{o}$ avanço é superestimado para um mesmo tempo, isto ocorre certamente devido ao maior perímetro molhado. Observa-se, também, que os perfis de infiltração obtidos com $\mathrm{P}_{1}, \mathrm{P}_{2}$ e $\mathrm{P}_{3}$, mostraram valores inferiores aos dados de campo, caso em que a recessão de campo é superior à estimada para os dados de Ramsey (1976). Para os dados de Karmeli (1979), no qual a recessão estimada se aproxima da recessão medida em campo, os perfis de infiltração calculados para $\mathrm{P}_{1}$ e $\mathrm{P}_{3}$ se aproxima aos dados de campo, mas o mesmo não ocorre para $\mathrm{P}_{2}$, uma vez que, a infiltração é função do tempo de oportunidade e consequentemente do tempo de avanço da água, e no caso de $\mathrm{P}_{2}$ este foi superestimado. Ante o exposto, conclui-se que o avanço e o perfil de infiltração calculados com $\mathrm{P}_{1}$ e $\mathrm{P}_{3}$, apresentam resultados semelhantes comparados aos valores de campos e aos obtidos com o modelo hidrodinâmico.

No cálculo do volume infiltrado, obtido com a Eq. 16, consideraram-se os três perímetros molhados, $\mathrm{P}_{1}, \mathrm{P}_{2}$ e $\mathrm{P}_{3}$, obtendo-se, respectivamente, $\mathrm{V}_{1}, \mathrm{~V}_{2}$ e $\mathrm{V}_{3} \mathrm{Na}$ Tabela 3, observa-se que, para o Experimento 1, o volume infiltrado $\mathrm{V}_{1}$ foi igual a $3,757 \mathrm{~m}^{3}$, enquanto o $V_{z}$ de Ramsey foi $4,230 \mathrm{~m}^{3}$, ou seja, $V_{z}$ é subestimado em aproximadamente $11 \%$. No Experimento 2, $\mathrm{V}_{1}$ foi $5,869 \mathrm{~m}^{3}$, enquanto $V_{z}$, calculado com o modelo hidrodinâmico foi $6,2 \mathrm{~m}^{3}$, sendo $\mathrm{V}_{\mathrm{z}}$ subestimado em aproximadamente $5,3 \%$, usando-se o modelo hidrodinâmico como padrão. Os valores de $\mathrm{V}_{2}$ nos primeiro e segundo experimentos foram de $6,488 \mathrm{e} 7,497 \mathrm{~m}^{3}$ respectivamente; neste caso, $\mathrm{V}_{\mathrm{z}}$ foi superestimado em aproximadamente $53,4 \%$ no primeiro e $21 \%$ no segundo, este último, tendo como referência o modelo hidrodinâmico. Para o volume calculado $\mathrm{V}_{3}$, os valores obtidos foram, respectivamente, 4,032 e $5,950 \mathrm{~m}^{3}$ para os primeiro e segundo experimentos, sendo $\mathrm{V}_{\mathrm{z}}$ subestimado em aproximadamente $4,7 \mathrm{e} 4,0 \%$, respectivamente, com relação aos dados de campo e ao modelo hidrodinâmico. 
Tabela 3. Volume infiltrado, $\mathrm{V}_{\mathrm{z}}, \mathrm{em} \mathrm{m}^{3}$, obtidos utilizando os perímetros $\mathrm{P}_{1}, \mathrm{P}_{2}, \mathrm{P}_{3}$, medido em campo e calculado pelo modelo hidrodinâmico

\begin{tabular}{lccccc}
\hline \multirow{2}{*}{ Experimentos } & $(1)$ & $(2)$ & $(3)$ & $(4)$ & $(5)$ \\
\cline { 2 - 6 } & 3,757 & 6,488 & 4,032 & 4,230 & - \\
\hline 1. Ramsey & 5,869 & 7,497 & 5,950 & - & 6,200 \\
2. Karmeli & Volume Infiltrado & $-\mathrm{V}_{\mathrm{z}}$ \\
\hline * (1) volume infiltrado calculado com $\mathrm{P}_{1}$, (2) volume infiltrado calculado com $\mathrm{P}_{2}$, (3) volume \\
$\begin{array}{l}\text { infiltrada calculado com } \mathrm{P}_{3} \text {, (4) volume infiltrado medido em campo, (5) volume infiltrado calculado } \\
\text { pelo modelo hidrodinâmico }\end{array}$
\end{tabular}

Ante o exposto, verifica-se que $\mathrm{P}_{1}$ representa bem o perímetro molhado na irrigação por sulco; no entanto, dentre os três valores $\mathrm{V}_{1}, \mathrm{~V}_{2}$ e $\mathrm{V}_{3}$ calculados, o que mais se aproximou dos dados de campo foi $\mathrm{o}_{3}$, calculado com o perímetro molhado estimado pela equação do arco da parábola. O que leva a admitir-se o uso da equação de $\mathrm{P}_{3}$, apresentada neste trabalho, como uma boa opção para o dimensionamento de sistemas de irrigação por sulco de forma parabólica e para avaliação dos mesmos.

Considerando-se, no Experimento 1, uma lâmina aplicada de $130 \mathrm{~mm}$ e se assumindo o perímetro molhado igual à superfície livre da água, $\mathrm{P}_{1}$, o modelo algébrico de Levien (1985) prediz eficiência de aplicação de água (Ea) igual a 22,6\% e eficiência de armazenamento (Es) de $28,9 \%$, enquanto no mesmo experimento, assumindo o perímetro molhado igual ao arco da parábola $\mathrm{P}_{3}$, obteve-se $\mathrm{Ea}=24,3 \%$ e $\mathrm{Es}=31 \%$. No segundo experimento, para uma lâmina aplicada de $100 \mathrm{~mm}$, para o perímetro molhado $\mathrm{P}_{1}$, o modelo algébrico prediz uma Ea igual a $60 \%$ e uma Es de $30 \%$; no entanto, considerando-se o perímetro molhado igual ao arco da parábola $\mathrm{P}_{3}$, o modelo algébrico prediz uma Ea igual a $61 \%$ e Es de $30,4 \%$, enquanto o modelo hidrodinâmico prediz Ea de $63 \%$ e Es de $31 \%$, usando o modelo hidrodinâmico como padrão. Verifica-se, mais uma vez, que a equação $\mathrm{P}_{3}$ representa bem o perímetro molhado na irrigação por sulco.

\section{CONCLUSÕES}

A análise desenvolvida neste trabalho possibilitou as seguintes conclusões:

1. Na irrigação por sulco, quando se utiliza a equação do arco da parábola para calcular o perímetro molhado, os valores estimados de avanço, recessão, lâmina d'água infiltrada e volume infiltrado, representam melhor a condição de campo se comparadas com as equações tradicionais.
2. A equação do arco da parábola pode ser utilizada para qualquer lâmina d'água, o que não ocorre com as demais equações de cálculo de perímetro molhado.

\section{LITERATURA CITADA}

Amim, S.L. Análise comparativa das técnicas de determinação das características de infiltração na irrigação por sulco. Fortaleza: Universidade Federal do Ceará, 1985. 136p. Dissertação Mestrado

Azevedo, C.A.V. de. Real - time solution of the inverse furrow advance problem. Logan: Universidade de Utah. S. Ed. 1992, 263p. Tese Doutorado

Azevedo, C.A.V. de. Modelagem em sistema de checagem e controle de irrigação por sulcos. In: Congresso Brasileiro de Engenharia Agrícola, 22, 1993, Anais Ilhéus: SBEA, 1993.

Carvalho, D.F. de; Oliveira, L.F.C. de; Pruski, F.F.; Soares, A.A.; Montovani, E.C. Influência do perfil geométrico do sulco no desempenho da irrigação. In: Congresso Brasileiro de Engenharia Agrícola, Viçosa, 1995. Resumo. SBEA. N.95-3-222.

Daker, A. Água na agricultura, manual de hidráulica agrícola, v.3. Irrigação e Drenagem. $5^{\text {a }}$ ed. Rio de Janeiro: Freitas Bastos, 1976. 453p.

Fok, Y.S. Principles and design of furrow irrigation systems. Lancaster, PA: Civil Publishing Co., Inc., 1988. 489-504p.

Karmeli, D.; Salazar, J.L.; Walker, W.R. Assessing the spatial variability of irrigation water applications. Fort Collins, USA: Department of Agricultural and Chemical Engineering. Colorado State University, 1979, 201p.

Levien, S.L, Desenvolvimento de um modelo matemático simplificado da irrigação superficial por sulcos abertos em declive. Fortaleza: Universidade Federal do Ceará, 1985. p.91 Dissertação Mestrado

Olitta, A.F. Os métodos de irrigação. $1^{a}$ ed. São Paulo: Universidade de São Paulo, 1978.267p.

Ramsey, M.K. Intake characteristics and flow resistance in irrigation furrows. Tucson: University of Arizona, 1976. 141p. Master Dissertation of Science

Scaloppi, E.J. Curso de elaboração de projetos de irrigação. $1^{\text {a }}$ ed. Brasília: PNI/Fundação CTH, 1986. T9.

SCS-USDA - Soil Conservation Service - United States Department of Agriculture. Furrow irrigations. Unedited Draft: University of California, 1980, 110p. Section 15. Chapter 5. National Engineering Handbook

Souza, F. Nonlinear hydrodynamic model of furrow irrigation. Davis: University of California. 1981. 172p. Tese Doutorado

Souza, F. de. Hidráulica da irrigação por superfície. DENA/UFC. 1983. 54p. Mimeografado 